\title{
El cambio en la sensibilidad a la insulina no altera el perfil de lípidos en mujeres con posmenopausia temprana
}

\author{
Ana Bertha Zavalza $\mathbf{G}^{1}$, Fernando Grover $\mathrm{P}^{1}$, José Miguel \\ Mora $\mathrm{M}^{2}$, Mayarí Centeno $\mathrm{L}^{1}$, Roberto Anaya $\mathrm{P}^{1}$. \\ No association between insulin \\ sensitivity and serum lipid levels \\ in postmenopausal women
}

Background: It is not clear if changes in serum lipid profile during menopause are related to changes in the distribution of fat or to insulin resistance. Aim: To look for an association between insulin resistance and changes in serum lipid levels in post menopausal women. Patients and methods: Cross-sectional study of 66 early postmenopausal women aged 45 to 55 years. Body mass index (BMI), waist-to hip ratio (WHR), total body adiposity, systolic blood pressure (SBP), diastolic blood pressure (DBP), mean blood pressure (MBP) and pulse pressure (PP) were measured. Total cholesterol, low-density lipoproteins, high-density lipoproteins, triglycerides, glucose and insulin were determined in a fasting blood sample. Insulin sensitivity was calculated using the homeostasis model assessment method (HOMA) and patients were stratified in quartiles according to this parameter. Results: Patients stratified in the different quartiles of insulin sensitivity were comparable in age and postmenopausal period, but had no differences in serum lipid levels. Compared to women in the lower quartile of insulin resistance, women in the upper quartile had higher BMI (23 and 27 $\mathrm{kg} / \mathrm{m}^{2}$ respectively, $\mathrm{p}=0.02$ ), SBP (110 and $125 \mathrm{mmHg}$ respectively, $\mathrm{p}<0.01$ ) and MBP (83 and $93 \mathrm{mmHg}$ respectively, $\mathrm{p}<0.01$ ). Differences in total body adiposity were observed between the lower, third, and the upper quartile $(30 \%, 35 \%$ and $36 \%$ respectively, $p<0.01)$. Conclusions: In this group of early postmenopausal women, no association was observed between the level of insulin sensitivity and serum lipid levels (Rev Méd Chile 2007; 135: 613-19).

(Key w ords: Hyperlipoproteinemias; Insulin resistance; Postmenopause)

Recibido el 3 de julio, 2006. Aceptado el 6 de noviembre, 2006.

${ }^{1}$ División de Educación e Investigación en Salud, Unidad Médica de Alta Especialidad, Hospital de Gíneco-Obstetricia, Centro Médico Nacional de Occidente, Instituto Mexicano del Seguro Social. ${ }^{2}$ Hospital General de Zona No. 14, Instituto Mexicano del Seguro Social. Guadalajara, México.

Correspondencia a: Fernando Grover Páez PhD Av. Gral. Eulogio Parra 3022, Col. Prados Providencia. CP 44670, Guadalajara Jal, México. Tel: 01 (33) 36-40-05-47 Fax: 01 (33) 36-40-05-47. E mail: fgroverp@hotmail.com 
$\mathrm{L}$ a mujer posmenopáusica es más resistente a la insulina que la premenopáusica. La edad, el incremento en la grasa corporal total, la adiposidad central, la deficiencia estrogénica, las alteraciones en el perfil lipídico y la homeostasis de la glucosa y la insulina son más frecuentes y favorecen la alta morbimortalidad cardiovascular después de la menopausia ${ }^{1}$. Godsland et $\mathrm{al}^{2}$, y Lindheim et $\mathrm{al}^{3}$, encontraron una correlación negativa entre la edad de la menopausia y la sensibilidad a la insulina, su metabolismo hepático o ambas. Brochu et $\mathrm{al}^{4}$, reportaron que en la mujer posmenopáusica el tejido adiposo visceral es un buen marcador de resistencia a la insulina (RI). Un incremento de 1 $\mathrm{mg} / \mathrm{dL}$ de colesterol de lipoproteínas de alta densidad (c-HDL) se asocia con una incidencia 3\% menor de enfermedad coronaria $^{5}$ y hasta $4,7 \%$ menos mortalidad en las mujeres ${ }^{6}$. La transición de la menopausia está marcada por cambios en el balance hormonal, con aumento de la adiposidad visceral los cuales se asocian con $\mathrm{RI}^{4}$. En la posmenopausia, también se presenta aumento del perfil de lípidos aterogénico, caracterizado por aumento del colesterol unido a lipoproteínas de baja densidad (c-LDL), de los triglicéridos (TG) y de las partículas pequeñas densas de LDL, con reducción de c-HDL y aumento de las concentraciones séricas de glucosa e insulina, tal vez como resultado directo de la falla ovárica o de manera indirecta a consecuencia de la redistribución central de la grasa corporal ${ }^{7-11}$. Los vínculos entre la resistencia a la insulina y la dislipidemia, hipertensión, hipercoagulación y aterosclerosis son numerosos y complejos. A nivel celular, basado en estudios in vitro, se ha observado que la incubación prolongada de células con altas concentraciones de insulina puede incrementar la secreción de lipoproteínas de muy baja densidad (VLDL), quizás debido al desarrollo de un estado crónico de RI en células después de un tratamiento prolongado. La resistencia a la entrada de glucosa a los tejidos dependiente de la estimulación por la insulina parece aumentar la actividad de la lipasa hepática. Estas VLDL enriquecidas en TG no constituyen precursores potenciales de las LDL y una alta proporción de ellas es convertida en remanentes de VLDL ricos en colesterol. Los cambios en las lipasas favorecen esta conversión ${ }^{6}$. Además, los niveles reducidos de c-HDL (10-20\% menores que en los controles) y apo A-1 se traducen en la existencia de menor cantidad de partículas HDL involucradas en el eflujo de colesterol desde los tejidos periféricos, el cual es el primer paso en el transporte reverso de colesterol. La menor cantidad de partículas HDL impide que estas ejerzan los múltiples efectos antiaterogénicos que se han descrito a nivel de la pared arterial, incluyendo su función como antioxidantes ${ }^{6}$. Actualmente no se cuenta con información suficiente que demuestre si las variaciones en el perfil lipídico dependen sólo de los cambios en el patrón de distribución de la grasa corporal, o si éstos se modifican dependiendo del nivel de resistencia a la insulina. El objetivo principal de este estudio fue identificar las modificaciones del perfil lipídico en relación al cambio en la sensibilidad a la insulina en mujeres posmenopáusicas.

\section{PACIENTES Y MÉTODOS}

Estudio transversal, analítico. Se incluyeron 68 mujeres con posmenopausia temprana y bioquímica $^{1}$, índice de masa corporal (IMC) $\leq 30 \mathrm{~kg} / \mathrm{m}^{2}$, con edades entre 45 y 55 años. Todas fueron seleccionadas de la misma comunidad y estrato socioeconómico. Ninguna era sedentaria o participaba en una actividad física intensa. No fumaban y su peso corporal se había mantenido estable 6 meses previos al estudio. No se encontraban bajo algún régimen de terapia hormonal de reemplazo 6 meses previos. No se incluyeron en el estudio, mujeres con antecedente personal de cáncer de mama o endometrio, de ooforectomía bilateral, presencia de tumoración mamaria palpable, con hipertensión arterial, enfermedad tromboembólica, cardiaca, hepática, renal o tiroidea, o que estuviesen tomando medicamentos que afectan el metabolismo de lípidos o carbohidratos.

Definición de posmenopausia. El estado de posmenopausia temprana se definió por el antecedente de un año a menos de 10 de amenorrea a partir del último periodo menstrual, así como una concentración de estradiol sérico menor de $39,5 \mathrm{pg} / \mathrm{ml}$ y una concentración sérica de hormona folículo estimulante (FSH) mayor de $67,0 \mathrm{mUI} / \mathrm{ml}^{1}$. En este grupo, el rango de años de posmenopausia fue de uno a diez, con un promedio de $6,5 \pm 2$.

Las concentraciones de estradiol sérico y de FSH se determinaron mediante inmunoensayo de 
electroquimioluminiscencia (Elecsys Systems 1010, Roche).

Formación de grupos. Las pacientes fueron estratificadas en cuartilas (17 por cuartil) de acuerdo a su nivel de sensibilidad a la insulina. RI: el índice de resistencia a la insulina fue calculado a partir de los valores iniciales de glucosa e insulina, siguiendo el modelo homeostático (HOMA) propuesto por Matthews y otros ${ }^{12}$, de acuerdo con la fórmula: RI= (insulina $x$ glucosa $) / 22,5$. La insulinemia se expresa en $\mu \mathrm{u} / \mathrm{mL}$ y la glucemia en $\mathrm{mmol} /$ L. Un valor bajo de HOMA-RI indica gran sensibilidad a la insulina, mientras que, un valor alto de HOMA-RI indica baja sensibilidad a la insulina (resistencia a la insulina).

Concentraciones de glucosa e insulina. La concentración de glucosa se midió por el método de glucosa oxidasa, usando un analizador automático (Boehringer Mannheim GMBH, Mannheim, Alemania). La insulina sérica fue determinada con un radioinmunoensayo con doble anticuerpo (RIA kit de insulina, Linco Research Inc., Charles, MO, USA). El coeficiente de variación intra e interensayo fue de $4 \%$ y $10 \%$, respectivamente.

Perfil de lípidos. La concentración de lípidos séricos (colesterol total, c-HDL y triglicéridos) se determinó por métodos enzimáticos. Las mediciones se realizaron con equipo automatizados. El cLDL se estimó por la fórmula de Friedewald ${ }^{15}$ : $(\mathrm{c}-\mathrm{LDL}=$ colesterol total-c-HDL - triglicéridos/5).

Mediciones antropométricas. La composición corporal (peso e índice de masa corporal) se determinó por bioimpedancia usando una báscula TANITA $^{\circledR}$ estacionaria (modelo 1990B, Valhalla Scientific Inc, San Diego, California) como se ha descrito previamente ${ }^{13,14}$. El sistema determina, mediciones de masa grasa y masa magra libre de grasa. El porcentaje de grasa corporal es calculado por dividir la masa grasa por el peso corporal. Estas mediciones han sido ya validadas contra mediciones de la grasa corporal total obtenida con métodos densitométricos hidrostáticos ${ }^{13,14}$.

El índice cintura/cadera (IC/C) se determinó con la voluntaria en bipedestación y con una cinta métrica; se consideró el diámetro de la cintura en el punto intermedio entre el margen de las últimas costillas y las crestas ilíacas y el diámetro de la cadera, tomando la circunferencia más amplia sobre los trocánteres, y para obtener el IC/C se estableció una relación entre los diámetros resultantes.

Presión arterial. La presión arterial se midió en el brazo izquierdo, después de un periodo de $5 \mathrm{~min}$ de descanso con la paciente en posición sentada. Las mediciones fueron realizadas con un esfigmomanómetro de mercurio por el mismo investigador, en el mismo brazo y la presión arterial sistólica (TAS) y diastólica (TAD) se registró en la fase I y V de Korotkof respectivamente.

Estadística. El tamaño de muestra estimado en 68 participantes se calculó mediante la fórmula para estudios transversales ${ }^{16}$, considerando la desviación estándar del promedio de las concentraciones de c-LDL en una población de mujeres posmenopáusicas ${ }^{11}$. Los valores se expresan en medianas y rangos. Para comparar las características clínicas y bioquímicas se utilizó un análisis de varianza de una vía con corrección de Tukey. Los datos fueron almacenados y analizados utilizando un software SPSS 10.0 para Windows (Inc., Chicago, IL).

La naturaleza y propósito del estudio fueron cuidadosamente explicados a cada participante antes de obtener su consentimiento voluntario por escrito. El protocolo de estudio fue revisado y aprobado por los Comités de Investigación y Ética del IMSS en Guadalajara, Jal, México.

\section{Resultados}

La sensibilidad a la insulina se estimó mediante el HOMA, el cual clasificó a las pacientes en cuatro niveles (cuartila inferior, segunda, tercera y superior), cuyos valores se expresan en promedios y desviación estándar (DE). La edad, y años de posmenopausia no fueron diferentes; el IMC, la cintura, la TAS y la presión arterial media (TAM), fueron más altos en la cuartila superior comparado con la inferior. En adición, el porcentaje de grasa corporal, fue menor en la cuartila inferior vs la tercera y la superior; y no se observaron cambios en la TAD y en la presión de pulso (Tabla 1).

Con relación a las concentraciones de los lípidos, de acuerdo al nivel de sensibilidad a la insulina, no se observaron cambios entre las cuartilas (Figura 1). 


\section{Tabla 1. C aracterísticas clínicas de acuerdo al nivel de sensibilidad a la insulina en mujeres con posmenopausia temprana}

\begin{tabular}{|lllll|}
\hline Variables & & \multicolumn{2}{c|}{ Cuartilas } \\
& Inferior $(\mathrm{n}=17)$ & Segunda $(\mathrm{n}=17)$ & Tercera $(\mathrm{n}=17)$ & Superior $(\mathrm{n}=17)$ \\
\hline HOMA & $1,09 \pm 0,26(0,6-1,5)$ & $1,96 \pm 0,26(1,6-2,4)$ & $3,13 \pm 0,49(2,5-4,0)$ & $5,81 \pm 1,80(4,1-10,5)$ \\
Edad (años) & $51(47-55)$ & $50(45-54)$ & $48(45-55)$ & $51(45-55)$ \\
Posmenopausia (años) & $5(1-10)$ & $4(1-10)$ & $2(1-10)$ & $6(1-10)$ \\
IMC (kg/m2) & $23(17-30)^{*}$ & $26(20-30)$ & $26(19-30)$ & $27(23-30)$ \\
Cintura (cm) & $79(70-102)^{* *}$ & $86(70-104)$ & $87(68-123)$ & $91(73-120)$ \\
Índice cintura cadera & $0,83(0,76-1,04)$ & $0,86(0,76-0,96)$ & $0,86(0,71-1,01)$ & $0,88(0,78-1,17)$ \\
TAS (mmHg) & $110(90-120)^{* * *}$ & $120(90-140)$ & $110(90-150)$ & $125(100-140)$ \\
TAD (mmHg) & $70(60-80)$ & $80(50-90)$ & $80(60-90)$ & $80(70-90)$ \\
TAM (mmHg) & $83(70-93)^{* * * *}$ & $90(67-107)$ & $90(70-110)$ & $93(80-107)$ \\
Presión de pulso (mmHg) & $40(30-50)$ & $40(20-65)$ & $40(25-60)$ & $45(30-70)$ \\
Grasa corporal total (\%) & $30(19-36)^{* * * * * *}$ & $32(23-43)$ & $35(25-44)$ & $36(28-42)$ \\
\hline
\end{tabular}

Mediana (rango). IMC=Índice de masa corporal, TAS=Tensión arterial sistólica, TAD=Tensión arterial diastólica, TAM=Tensión arterial media. Prueba ANOVA.

${ }^{*}$ p 0,01 vs cuartila superior. ${ }^{* *}$ p 0,05 vs cuartila superior. ${ }^{* * *}$ p 0,007 vs cuartila superior. ${ }^{* * * *}$ p 0,03 vs cuartila superior. ${ }^{* * * * * *} \mathrm{p}<0,01$ vs cuartilas tercera y superior.

\section{DisCUSIÓN}

Diversos estudios apoyan la hipótesis de que la mujer posmenopáusica desarrolla RI y presenta con mayor frecuencia alteraciones en el perfil de lípidos, así como, presión arterial, IMC y porcentaje de grasa corporal, comparado con premenopáusicas de la misma edad ${ }^{4}$. Se ha demostrado, que las principales alteraciones en el perfil de lípidos se manifiestan por un nivel reducido de c-HDL e incremento de triglicéridos y c-LDL, aunque en mujeres mayores de 50 años valores elevados de CT también se han identificado $^{6,8,11}$. Aunque la asociación entre la adiposidad abdominal y las anormalidades de los lípidos es bien conocida, los fundamentos fisiopatológicos no se han aclarado en su totalidad; estos cambios suelen estar vinculados al aumento de peso corporal y distribución central de la grasa que generan resistencia a la insulina con incremento de ácidos grasos libres (AGL) circulantes, reducción de adiponectina y elevación de las concentraciones de lipoproteín-lipasa en el tejido adiposo y de la lipasa hepática, habi- tualmente desencadenados por la deficiencia estrogénica $^{17}$. Esta asociación entre la resistencia a la insulina y el incremento en el IMC, así como con la distribución central de la grasa, se confirma con la presente investigación; sin embargo, a pesar de que existe un incremento no significativo en los niveles de triglicéridos, las concentraciones de CT, c-HDL, c-LDL no se modificaron, ya que como se ha reportado ${ }^{17}$, los cambios en relación a los lípidos, suelen ser mayores en el periodo perimenopáusico, en donde la magnitud de estas variaciones se debe probablemente a las determinantes diferenciales del cambio en los factores de riesgo, como la reducción en los niveles de estradiol, ganancia de peso, e incremento en la circunferencia de la cintura durante la perimenopausia (periodo que comprende de tres a ocho años antes y uno después de la menopausia) ${ }^{1}$. Los mecanismos responsables para incrementar las concentraciones de colesterol en la posmenopausia temprana son inciertos. Como se ha reportado en estudios epidemiológicos ${ }^{18}$, en el presente estudio, observamos que más que una asociación 


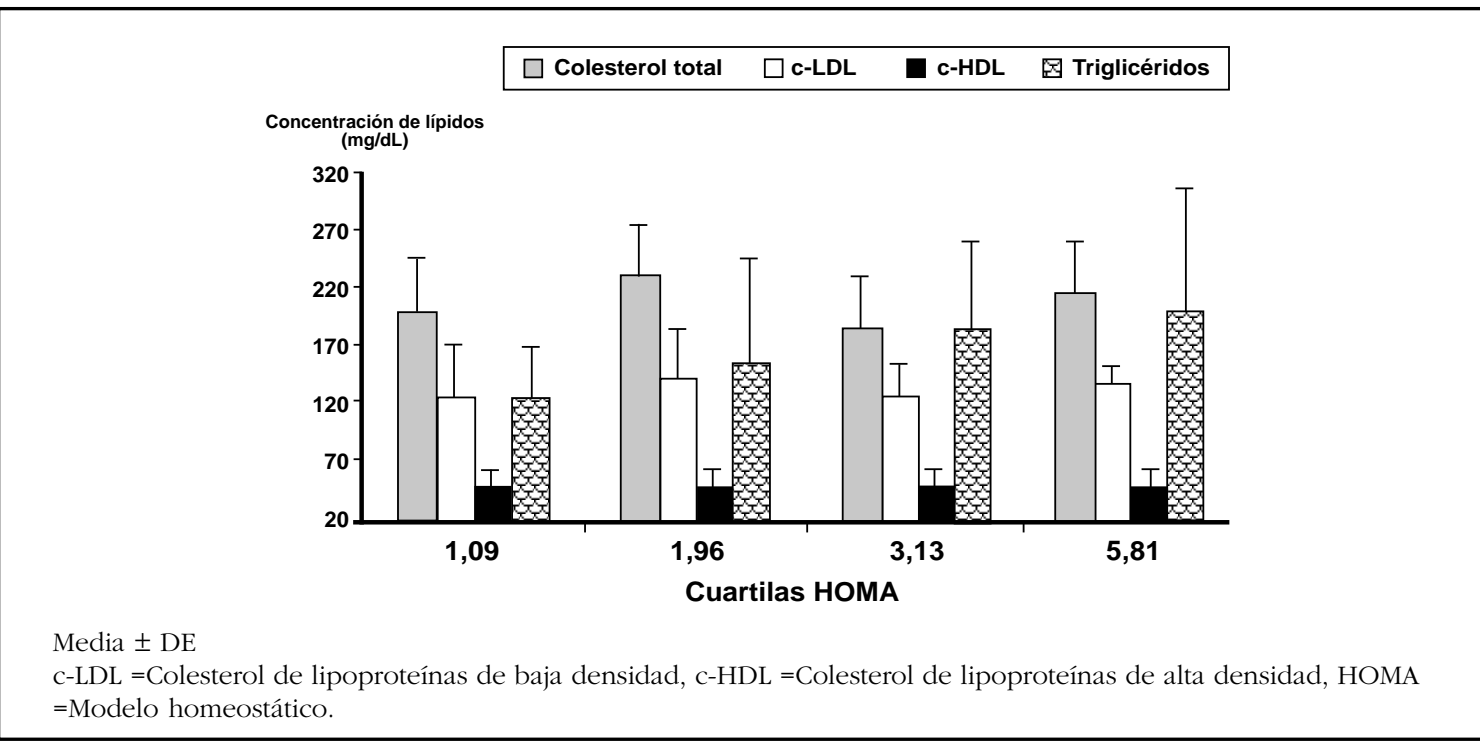

Figura 1. Promedio de las concentraciones de colesterol total, c-LDL, c-HDL y triglicéridos de acuerdo a la cuartila de sensibilidad a la insulina en mujeres con posmenopausia temprana.

directa entre las alteraciones del perfil de lípidos a la resistencia a la insulina, influye significativamente el índice de masa corporal y porcentaje de grasa. Las concentraciones de estradiol son un factor importante que se correlaciona negativamente tanto con el colesterol total como el de baja densidad ${ }^{19}$, ya que los estrógenos juegan un papel importante independiente en la regulación del metabolismo de los lípidos en las mujeres; es decir, estos inducen cambios favorables en el perfil de lipoproteínas plasmáticas en la premenopausia, tales como, un incremento de la concentración de c-HDL en particular la sub-fracción $\mathrm{HDL}_{2}$ y porcentaje de síntesis de Apo-A, además, favorecen la reducción en los niveles de c-LDL y sobre-regula el receptor de $\mathrm{LDL}^{6,8,11}$. Estos efectos pueden desaparecer parcialmente durante la menopausia debido a la reducción en la producción, concentración sanguínea y liberación tisular de estrógenos.

Por otra parte, los cambios en la presión sistólica, presión de pulso y glucosa de ayuno se alteran con mayor frecuencia durante la posmenopausia, debido al efecto del envejecimiento, con aumento en la rigidez vascular y en la proporción de grasa corporal ${ }^{4}$. En nuestro estudio, la edad promedio de las pacientes fue de 50 años y la diferencia de ésta entre las cuartilas no fue significativa; esto apoya claramente estas dos hipótesis; primero, el impacto de la resistencia a la insulina sobre los lípidos no se demostró, ya que las pacientes no se encontraban en el periodo perimenopáusico y no presentaban otras determinantes de riesgo como edad avanzada u obesidad. Segundo, la rigidez vascular identificada mediante la elevación en las cifras de TAS y TAM observada en la presente investigación demuestra la asociación que existe entre la RI y el proceso de envejecimiento del sistema vascular que se acelera durante un periodo rápido de deficiencia estrogénica $^{4}$. Por otra parte, se ha demostrado que la hipertensión arterial y los niveles altos de insulina están positivamente correlacionados. Se sabe que la hiperinsulinemia puede dar como resultado un incremento de la reabsorción de sodio y agua por las células tubulares del riñón ${ }^{10}$ y esto puede estar asociada con la hipertensión volumen-dependiente. Sin embargo, no está claro con qué frecuencia la hipertensión volumen-dependiente está presente en individuos insulino-resistentes y pacientes con diabetes. La asociación entre la resistencia a la 
insulina y la hipertensión no es tan fuerte como su asociación con la dislipidemia; solo alrededor de $50 \%$ de los individuos hipertensos son insulino-resistentes $6,10,19$. Se ha sugerido que las anomalías en la vasodilatación y el flujo sanguíneo constituyen un vínculo entre la hipertensión y la resistencia a la insulina.

Cabe mencionar, que este estudio tiene limitaciones en relación al método con el que medimos la sensibilidad a la insulina, ya que no es el estándar de oro $^{20}$, sin embargo, el modelo homeostático es un método alternativo, simple y barato y se ha utilizado ampliamente para expresar la resistencia a la insulina en diversas poblaciones en donde son impracticables los métodos de referencia ${ }^{12}$.

\section{REFERENCIAS}

1. Van Der Schouw YT, Van Der Graaf Y, Steyerberg EW, Marinus JCE, Jan Dirk B. Age at menopause as a risk factor for cardiovascular mortality. The Lancet 1996; 347: 714-8.

2. Godsland IF, Crook D, Stevenson JC, Collins P, Rosano GMC, LeEs B ET AL. The insulin resistance syndrome in postmenopausal women with cardiological syndrome X. Br Heart J 1995; 74: 47-52.

3. Lindheim SR, Presser SC, Ditkoff EC, Vijod MA, Stanczyk FZ, Lobo RA. A possible bimodal effect of estrogen on insulin sensitivity in postmenopausal women and the attenuating effect of added progestin. Fertil Steril 1993; 60: 664-7.

4. Brochu M, Starling RD, Tchernof A, Matthews DE, García-Rubi E, Poehlman ET. Visceral adipose tissue is an independent correlate of glucose disposal in older obese postmenopausal women. J Clin Endocrinol Metab 2000; 85: 2378-84.

5. Castelli WP. Framingham, Massachusetts. Epidemiology of Coronary Heart Disease: The Framingham Study. Am J Med 1984; 27: 4-12.

6. LEWIS GF. Fatty acid regulation of very low density lipoprotein (VLDL) production. Curr Opin Lipidol 1997; 8: 146-53.
En conclusión, nuestro estudio demuestra que el nivel de sensibilidad a la insulina no afecta de manera negativa las concentraciones de colesterol total, c-LDL, c-HDL, ni los triglicéridos cuando la mujer ha rebasado el periodo perimenopáusico y son la obesidad total y central las responsables de las alteraciones lipídicas después de ésta etapa. Por otra parte, el método de selección y el tamaño del grupo de referencia en este estudio no aseguran la representatividad del mismo, por lo que los valores que se presentan sólo constituyen un punto de inicio para estudios posteriores que evalúen su utilidad, en términos de su correlación con métodos de referencia como los antes señalados, o por su valor predictivo del desarrollo de diabetes o sus complicaciones en poblaciones de riesgo.

7. Carr MC. The Emergence of the Metabolic Syndrome with Menopause. J Clin Endocrinol Metab 2003; 88: 2404-11.

8. Poehlman ET, Toth MJ, Ades PA, Rosen CJ. Menopause-associated changes in plasma lipids, insulinlike growth factor I and blood pressure: a longitudinal study. Eur J Clin Invest 1997; 27: 3226.

9. Matthews KA, Meilahn E, Kuller LH, Kelsey SF, Caggiula AW, Wing RR. Menopause and risk factors for coronary heart disease. N Engl J Med 1989; 321: 641-6.

10. Austin M, King M-C, Vranizan K, Newman B, Krauss R. Inheritance of low density lipoprotein subclass patterns: results of complex segregation analysis. Am J Hum Genet 1988; 43: 838-46.

11. Carr MC, Kim KH, Zambon A, Mitchell ES, Woods NF, Casazza CP ET al. Changes in LDL density across the menopausal transition. J Invest Med 2000; 48: 245-50.

12. Matthews DR, Hosker JP, Rudenski AS, Naylor BA, Treacher DF, Turner RC. Homeostasis model assessment: insulin resistance and beta-cell function from fasting plasma glucose and insulin concentrations in man. Diabetologia 1985; 28: 412-9. 
13. Lukaski HC, Johnson PE, Bolonchuck WW, Likken GI. Assessment of fat-free mass using bioelectrical impedance measurements of the human body. Am J Clin Nutr 1985; 41: 810-7.

14. Kushner RF. Bioelectrical impedance analysis: a review of principles and applications. J Am Coll Nutr 1992; 11: 199-209.

15. Friedewald WT, Levy RI, Fredrickson DS. Estimation of the concentration of low-density lipoprotein cholesterol in plasma without use of preparative ultracentrifuge. Clin Chem 1972; 18: 499-502.

16. Jeyaseelan L, RaO PSS. Methods of determining sample sizes in clinical traials. Indian Ped 1989; 26: 115-21.

17. Boden G. Pathogenesis of type 2 diabetes. Insulin resistance. Endocrinol Metab Clin North Am 2001; 30: 801-15.

18. Matthews KA, Kuller LH, Sutton-Tyrrrll K, Chang YF. Changes in cardiovascular risk factors during the perimenopause and postmenopause and carotid artery atherosclerosis in healthy women. Stroke 2001; 32: 1104-11.

19. Jenner JL, Ordovas JM, Lamon-Fava S, Schaefer MM, Wilson PVF, Castelli WP ET al. Effects of age, sex, and menopausal status on plasma lipoprotein (a) levels. The Framingham Offspring Study. Circulation 1993; 87: 1135-41.

20. De Fronzo RA, Tobin JD, Andres R. Glucose clamp technique: a method for quantifying insulin secretion and resistance. Am J Physiol 1979; 237: E214-E223.

\section{Agradecimientos}

Al personal del laboratorio de la Unidad de Medicina Familiar $\mathrm{N}^{\mathrm{O}} 88$ del IMSS. 